\title{
Neonatal thrombocytopenia: causes and management
}

\author{
I Roberts, N A Murray
}

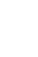

Neonatal thrombocytopenia is a common clinical problem. Thrombocytopenia presenting in the first 72 hours of life is usually secondary to placental insufficiency and caused by reduced platelet production; fortunately most episodes are mild or moderate and resolve spontaneously. Thrombocytopenia presenting after 72 hours of age is usually secondary to sepsis or necrotising enterocolitis and is usually more severe and prolonged. Platelet transfusion remains the only treatment. There is a need for trials to define the safe lower limit for platelet count and which neonates will benefit from treatment.

See end of article for authors' affiliations ......................

Correspondence to: Dr Roberts, Department of Haematology,

Hammersmith Campus,

Hammersmith Hospital, Du

Cane Road, London

W12 ONN, UK;

irene.roberts@ic.ac.uk

Accepted

4 October 2002 mon in neonatal intensive care unit ( this approach now needs to be re-evaluated. when clearly clinically indicated. right answer here". rary management plans for each.

\section{DEFINITION}

Arch Dis Child Fetal Neonatal Ed 2003;88:F359-F364

\section{A common clinical problem?}

Twenty six weeks gestation, day 4, respiratory distress syndrome, stable on mechanical ventilation; platelet count $85 \times 10^{9} / \mathrm{I}$ and falling.

Platelet count normal or abnormal? Abnormal. Common or uncommon clinical problem? Common.

Thrombocytopenia requires specific treatment? Depends on the concurrent circumstances.

Reason for thrombocytopenia? Simple clinical and laboratory evaluation provides the answer in most newborns.

platelet count of all healthy newborn infants, regardless of gestational age, should be $150 \times 10^{\circ} / 1$ and above, and counts below this represent thrombocytopenia, just as in older children and adults.

\section{INCIDENCE}

\section{At birth}

Thrombocytopenia is present in $1-5 \%$ of newborns at birth, ${ }^{7-9}$ and severe thrombocytopenia (platelets $<50 \times 10^{9} / 1$ ) occurs in $0.1-0.5 \% .^{9-13}$ patients that it may be tempting to disregard it as a clinical problem and simply resort to platelet transfusion when the count gets "too low for comfort". However, there are several reasons why

Platelets for transfusion are associated with risks ${ }^{1}$ and are likely to be in short supply because of both increased demand and measures to reduce the risk of possible variant CreutzfeldtJakob disease ${ }^{2}$ and should therefore only be given

The main causes of neonatal thrombocytopenia are now becoming clearer and many traditionally held beliefs have recently been shown to have little or no evidence to support them. Further defining the causes and mechanisms behind the common forms is the only way to develop more appropriate treatment, including novel approaches. We owe it to our vulnerable patients and juniors to stop saying “we simply don't know the

This paper will first briefly review the definition and incidence of neonatal thrombocytopenia, then describe current ideas about the causes of the common and clinically important forms and its clinical impact, and finally present contempo-

A number of large studies have shown that the fetal platelet count is above $150 \times 10^{9} / \mathrm{l}$ by the second trimester of pregnancy, ${ }^{3-6}$ and then remains fairly constant until term. ${ }^{7}$ Therefore the normal

\section{In NICU patients}

Thrombocytopenia develops in 22-35\% of all babies admitted to NICUs ${ }^{14-16}$ and in up to $50 \%$ of those admitted to NICUs who require intensive care. ${ }^{15}{ }^{16}$ A considerable proportion (20\%) of these episodes of thrombocytopenia are severe. ${ }^{14} 17$ This means that $8 \%$ of preterm and $6 \%$ of all neonates admitted to an NICU have severe thrombocytopenia ${ }^{17}$ and are at increased risk of haemorrhage, presenting a common management problem.

\section{CAUSES AND MECHANISMS}

It is well recognised that many fetomaternal and neonatal conditions are associated with thrombocytopenia. ${ }^{18-20}$ Previous detailed studies have attempted to define the mechanisms by which these conditions cause thrombocytopenia, ${ }^{14} 15$ but, until recently, the mechanism underlying many neonatal thrombocytopenias remained unknown. As a result, classifications based on mechanism have proved of little practical help to neonatal paediatricians because of overemphasis of rare conditions of

Abbreviations: NICU, neonatal intensive care unit; NEC, necrotising enterocolitis; NAITP, neonatal alloimmune; thrombocytopenia; HPA, human platelet antigen; $\mathrm{ICH}$, intracranial haemorrhage; IVIG, intravenous immunoglobulin; Tpo, thrombopoietin; IL, interleukin; rh, recombinant human 
Table 1 Classification of fetal and neonatal thrombocytopenias

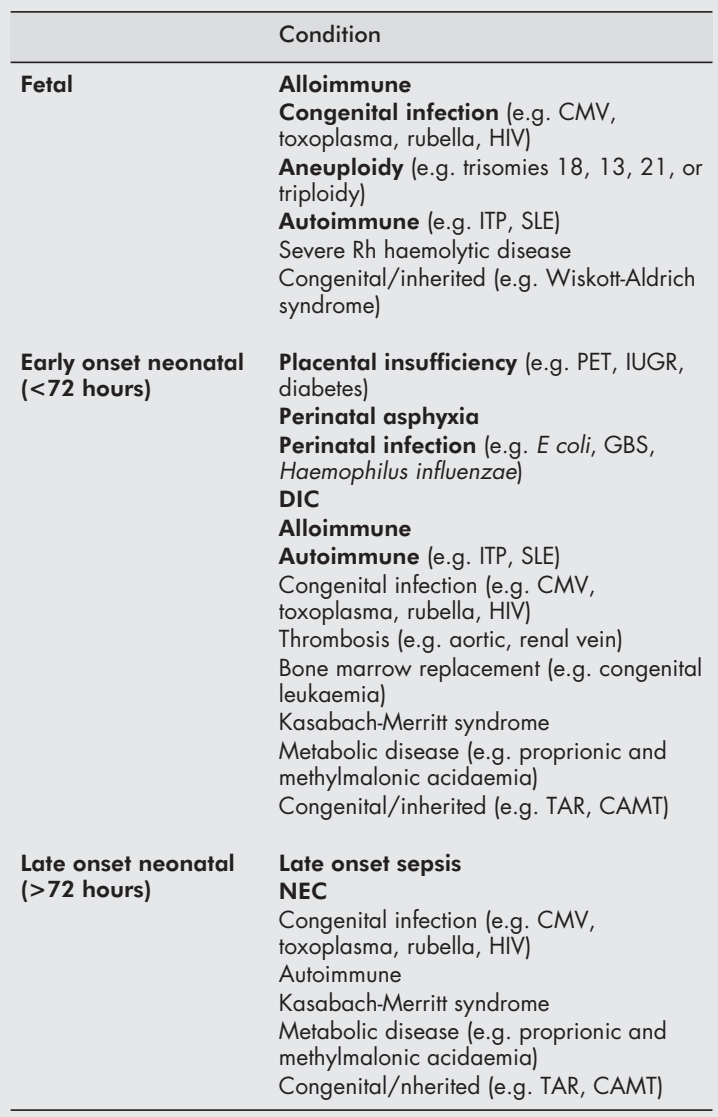

The most common conditions are highlighted.

CMV, Cytomegalovirus; ITP, idiopathic thrombocytopenic purpura SLE, systemic lupus erythematosus; PET, pre-eclampsia; IUGR, intrauterine growth restriction; $E$ coli Escherichia coli; GBS, group B streptococcus; DIC, disseminated intravascular coagulation; TAR, thrombocytopenia with absent radii; CAMT, congenital amegakaryocytic thrombocytopenia; NEC, necrotising enterocolitis.

known mechanism-for example, thrombocytopenia with absent radii (TAR syndrome; see table 1 for "practical" classification). They have also tended to perpetuate widely held beliefs that common neonatal conditions-for example, sepsis-cause thrombocytopenia because of platelet consumption due to disseminated intravascular coagulation, despite the fact that there is very little evidence to support this. ${ }^{14}{ }^{15}$ Although understanding the mechanisms underlying neonatal thrombocytopenia may seem to be an academic exercise, we believe it is only by clearly defining these mechanisms that more rational and innovative treatments can be developed.

\section{Impaired platelet production}

Recent work by ourselves and others shows that the major mechanism underlying neonatal thrombocytopenia is impaired platelet production. In $75 \%$ of all cases, the low platelet count is either present at birth or develops by 72 hours of life. ${ }^{14}{ }^{16}$ Only a minority of these patients have immunological disorders or coagulopathy causing thrombocytopenia (see below). Most of the remaining patients are preterm neonates born after pregnancies complicated by placental insufficiency and/or fetal hypoxia-for example, maternal pre-eclampsia and fetal intrauterine growth restriction. Neonates with this early onset thrombocytopenia (table l) have impaired megakaryocytopoiesis and platelet production; megakaryocytes and their precursor and progenitor cells are considerably reduced at birth, ${ }^{162122}$ and levels of the megakaryocytopoietic cytokine thrombopoietin (Tpo) are therefore elevated. ${ }^{23}$

\section{Consumption and sequestration}

Increased platelet consumption and/or sequestration are the major mechanisms in about $25-35 \%$ of episodes of neonatal thrombocytopenia. Overall, $15-20 \%$ of neonatal thrombocytopenias present at birth result from transplacental passage of maternal platelet alloantibodies and autoantibodies, ${ }^{912}$ and disseminated intravascular coagulation is responsible for a further $10-15 \%$ of cases, nearly always in babies who are very ill, particularly in association with perinatal asphyxia and infection. ${ }^{14} 15$ Thrombosis or platelet activation/ immobilisation at sites of inflammation-for example, in the gut during necrotising enterocolitis (NEC) or in haemangiomas-are further examples of neonatal thrombocytopenias principally caused by platelet consumption. In addition, there is evidence that limited splenic sequestration of platelets occurs in sick newborns. ${ }^{24}$

\section{Combined mechanisms}

Many neonates develop thrombocytopenia as a result of multiple mechanisms. A preterm neonate from a mother with pre-eclampsia who develops early bacterial sepsis and a baby with intrauterine growth restriction who develops NEC may both become thrombocytopenic as a result of underlying impaired platelet production (after pre-eclampsia or intrauterine growth restriction) combined with platelet consumption (during sepsis or NEC). Indeed it is likely that most neonates who develop thrombocytopenia do so because their adverse fetal environment causes impaired megakaryocytopoiesis at birth, with a predisposition for thrombocytopenia to worsen when the baby is exposed to concurrent neonatal platelet consumptive "stress". In addition, during sepsis and NEC, the natural history of thrombocytopenia (rapid onset and progression followed by slow recovery over five to seven days) suggests that it probably results from a combination of mechanisms - that is, platelet consumption (rapid onset phase) followed by impaired platelet production (slow recovery phase).

\section{COMMON PRESENTATIONS OF THROMBOCYTOPENIA IN THE PERINATAL PERIOD Fetal thrombocytopenia}

Fetal thrombocytopenia is now identified more often, as a consequence of primary diagnostic investigations for inherited thrombocytopenia (such as Wiskott-Aldrich syndrome (table 2$)^{6}$ or alloimmune thrombocytopenia in fetuses with intracranial haemorrhage, ${ }^{25}$ or during assessment of fetuses with ultrasound evidence-for example, hydrops-of congenital infections, haemolytic disease, or aneuploidy. ${ }^{7}$

\section{Thrombocytopenia in an otherwise healthy term baby}

Although an otherwise healthy term baby presenting with haemorrhage, purpura, or other signs of severe thrombocytopenia is uncommon, it may represent a neonatal emergency in order to avoid the risk of severe haemorrhage. The usual cause of this situation is neonatal alloimmune or autoimmune thrombocytopenia, although rare congenital or inherited thrombocytopenias may present in the neonatal period with haemorrhage (table 2).

\section{Neonatal alloimmune thrombocytopenia (NAITP)}

NAITP, the platelet equivalent of haemolytic disease of the newborn, is caused by transplacental passage of maternal alloantibodies directed against fetal platelet antigens inherited from the father but absent on maternal platelets. The most commonly detected antibodies are those directed against human platelet antigen (HPA)-la and HPA-5b, which are responsible for $80 \%$ and $10-15 \%$ of cases respectively. ${ }^{26-28}$ 
Table 2 Congenital and inherited thrombocytopenias that may present in the fetus or neonate

\begin{tabular}{ll}
\hline $\begin{array}{l}\text { Thrombocytopenia (with } \\
\text { abnormal platelet function) }\end{array}$ & $\begin{array}{l}\text { Bernard-Soulier syndrome } \\
\text { Wiskott-Aldrich syndrome } \\
\text { X-linked thrombocytopenia } \\
\text { Chediak-Higashi syndrome } \\
\text { Quebec platelet disorder } \\
\text { Some giant platelet syndromes (e.g. } \\
\text { Montreal syndrome) }\end{array}$ \\
Thrombocytopenia (without & $\begin{array}{l}\text { Fanconi's anaemia } \\
\text { marked thrombocytopathy) } \\
\text { TAR syndrome } \\
\text { Amegakaryocytic thrombocytopenia } \\
\text { Giant platelet syndromes (e.g. } \\
\text { May-Hegglin anomaly, Sebastian } \\
\text { syndrome, Fechtner syndrome) } \\
\text { Autosomal dominant thrombocytopenia }\end{array}$ \\
\hline
\end{tabular}

See Roberts and Murray ${ }^{79}$ for a full review of these conditions. TAR, Thrombocytopenia with absent radii.

However, severe NAITP has also recently been reported in association with anti-HPA- $3 \mathrm{a}^{29}$ and maternal HLA antibodies either alone ${ }^{3031}$ or in combination with HPA-la antibodies. ${ }^{32}$

The incidence of clinically apparent NAITP is about one in 1500 pregnancies, ${ }^{25}{ }^{27}$ although data from Ireland suggest that many neonatal cases remain undiagnosed. ${ }^{28}$ The disease varies in severity from mild to moderate resolving in the first week of life without clinical sequelae to extensive fetal or neonatal intracranial haemorrhage ( $\mathrm{ICH}$ ) leading to death or long term neurodevelopmental sequelae..$^{25-27}{ }^{33}$ In untreated cases, ICH occurs in about $10 \%$ of cases, with long term neurodevelopmental sequelae in $20 \%$ of survivors. ${ }^{25}{ }^{26}$ Unlike haemolytic disease, severe NAITP occurs during the first pregnancy in $40-50 \%$ of cases. The diagnosis depends on demonstrating platelet antigen incompatibility between mother and fetus/ baby; in up to $10 \%$ of cases antibodies to the platelets are undetectable during pregnancy and can only be found after delivery. ${ }^{25}{ }^{34}$

\section{Antenatal treatment}

Antenatal treatment for NAITP remains controversial. ${ }^{35-41}$ The options are:

- fetal blood sampling with intrauterine platelet transfusion for thrombocytopenic fetuses;

- fetal blood sampling with maternal intravenous immunoglobulin (IVIG) treatment if the fetus is thrombocytopenic;

- "tailored" approach reserving fetal blood sampling and intrauterine transfusion for cases in which a previous sibling suffered an ICH.

In the United Kingdom, it has been common practice in pregnancies with a previously severely affected baby to recommend fetal blood sampling from 24 weeks gestation to determine the fetal platelet count followed by weekly transfusions of thrombocytopenic fetuses with HPA compatible platelets until delivery to coincide with lung maturity (32-34 weeks). ${ }^{42}{ }^{43}$ Recent data show that, although procedure related fetal mortality is low, the rate of fetal loss per pregnancy is fairly high $(8.3 \%)$ because of the need for weekly fetal platelet transfusions to maintain the fetal platelet count. ${ }^{43}$ An alternative, less invasive approach is fetal platelet count monitoring together with maternal IVIG treatment if the fetus is thrombocytopenic. Maternal IVIG stabilises the fetal platelet count in up to $75 \%$ of cases, ${ }^{44}$ although some studies have reported a much lower response rate. ${ }^{45} 46$ Radder et al ${ }^{36}$ have recently suggested a combined approach in which "aggressive" treatment (fetal platelet count monitoring and intrauterine platelet transfusion combined with maternal IVIG) was reserved for cases with a previously affected sibling with an ICH; pregnancies in which the previous siblings had no ICH were managed with maternal IVIG only, with no fetal platelet count monitoring. The good results in both treatment arms in this report suggest that this approach may be a useful compromise, as only those fetuses with the highest risk of haemorrhage are exposed to the risks of intrauterine therapy.

\section{Neonatal treatment}

All severely affected neonates (ICH or other serious haemorrhage and/or platelet count $<30 \times 10^{9} / 1$ ) should receive HPA compatible platelets from either accredited HPA typed donors or from the mother (after washing to remove antibody). ${ }^{38}$ If both sources of compatible platelets are unavailable, IVIG ( 1 $\mathrm{g} / \mathrm{kg} /$ day on two consecutive days or $0.5 \mathrm{~g} / \mathrm{kg} /$ day for four days) is also effective in raising the platelet count, ${ }^{47}$ although the rise may be delayed for $24-48$ hours. ${ }^{48}$ IVIG can be combined with random donor platelet transfusion, as this may achieve a transient rise in platelet count until IVIG itself becomes effective ${ }^{47}$ The aim should be to maintain the platelet count at $>30 \times 10^{9} / \mathrm{l}$ for the first week of life or for as long as there is evidence of continuing haemorrhage. In suspected cases of NAITP, babies with severe thrombocytopenia should be transfused with donor platelets that are both HPA-la and HPA-5b negative ${ }^{49}{ }^{50}$ until the diagnosis is established.

\section{Neonatal autoimmune thrombocytopenia}

Transplacental passage of maternal platelet autoantibodies in mothers with idiopathic thrombocytopenic purpura or systemic lupus erythematosus may cause neonatal autoimmune thrombocytopenia in about $10 \%$ of cases. ${ }^{51}$ Maternal disease severity and/or platelet count during pregnancy can be used to predict the neonatal platelet count in most cases, but the clinical manifestations are less severe than in NAITP, and the risk of ICH is $1 \%$ or less. ${ }^{52}$ However, all neonates of mothers with autoimmune disease should have a cord blood platelet count determined at birth and again at 24 hours. $^{53}$ In thrombocytopenic neonates, the platelet count should be repeated daily for the next three to four days, as platelet counts are commonly at their lowest during this time before rising spontaneously by day 7 in most cases. ${ }^{54}$ As most babies found to have an ICH secondary to maternal autoimmune disorders have had platelet counts of $<30 \times 10^{9} / \mathrm{l}$, it is common practice to treat any neonates with severe thrombocytopenia (platelets $<30 \times 10^{9} / 1$ ) with IVIG regardless of whether or not there is evidence of bleeding..$^{53}$ There is no clear evidence that this approach is of benefit or that the threshold level of $30 \times 10^{9} / 1$ is appropriate; however, IVIG at a dose of 1 $\mathrm{g} / \mathrm{kg} /$ day on two consecutive days or $0.5 \mathrm{~g} / \mathrm{kg} /$ day for four days is usually effective in raising the platelet count. It is not uncommon for a second course of IVIG to be required two to three weeks after birth if the platelet count again falls below $(20-30) \times 10^{9} / 1$ because of persistence of the maternal platelet antibodies.

\section{Thrombocytopenia in NICU patients}

\section{Early onset thrombocytopenia}

Most cases of thrombocytopenia in babies admitted to NICUs are discovered "incidentally". The majority are preterm neonates and most (75-90\%) will develop early onset thrombocytopenia because of placental insufficiency/fetal hypoxia. ${ }^{16}{ }^{23}$ This thrombocytopenia has a remarkably consistent pattern (table 3 ), with a platelet nadir around day 4 and resolution by $7-10$ days of life ${ }^{14}{ }^{16}$; precipitous falls in platelet count are uncommon, and the platelet nadir rarely falls below $50 \times 10^{9} / \mathrm{l}$. Thus the most common pattern of thrombocytopenia in the newborn is predictable, evolves slowly, and, in the majority, is a "benign" phenomenon. By contrast, severe early thrombocytopenia (platelets $<50 \times 10^{9} / 1$ ) at $<72$ hours of life is much less common and occurs in term as well as preterm infants. The usual causes are severe perinatal infections-for 
Table 3 Comparison of natural history of early and late onset thrombocytopenia in neonates

\begin{tabular}{ll}
\hline Early & Late \\
\hline Mild to moderate & Severe \\
(platelet nadir rarely $\left.<50 \times 10^{9} / I\right)$ & (platelet nadir frequently $<50 \times 10^{9} /$ I) \\
Evolves slowly over several days & Rapid onset and progression over $24-48$ hours \\
Associated with: & Associated with: \\
Complicated pregnancies (PET, IUGR, maternal diabetes) & Sepsis and NEC \\
Rarely requires specific treatment & Multiple platelet transfusions often required \\
Mechanism: & Mechanism: \\
Impaired platelet production & Combined platelet consumption and impaired production \\
\hline
\end{tabular}

PET, Pre-eclampsia; IUGR, intrauterine growth restriction; NEC, necrotising enterocolitis.

example, group B streptococcus, Escherichia coli, and Listeria monocytogenes - and after perinatal asphyxia, particularly in term neonates. In both situations, disseminated intravascular coagulation is often a significant contributor.

\section{Late onset thrombocytopenia}

Our recent work ${ }^{17}$ clearly shows that thrombocytopenia developing, or significantly worsening, at $>72$ hours is almost exclusively caused by late onset sepsis or NEC. This late onset thrombocytopenia has a distinct natural history (table 3). Usually thrombocytopenia develops with the early signs of sepsis or NEC and often progresses rapidly, with a platelet nadir reached within $24-48$ hours. ${ }^{17}$ Thrombocytopenia is often severe, with affected neonates often requiring platelet transfusions until sepsis or NEC are controlled, followed by a slow recovery in platelet numbers over the following four to five days. ${ }^{17}$ Affected neonates are often profoundly sick, require intensive care, and in our unit have a $10-15 \%$ mortality. ${ }^{17}$

\section{MANAGEMENT}

\section{Platelet transfusion}

As yet, there are no widely accepted guidelines for platelet transfusion in newborns with non-immunologically mediated thrombocytopenia. ${ }^{19556}$ However, as the evidence that platelet transfusion improves neonatal outcome is lacking (see below), recent guidelines are more conservative than previous recommendations (table 4 ). ${ }^{57}$ As a general rule platelets should be given to thrombocytopenic neonates when the degree of thrombocytopenia alone or in combination with other complications results in an unacceptable risk of haemorrhage, and they should be given in tandem with aggressive therapy for the underlying conditions precipitating the thrombocytopenia. The risk of haemorrhage is difficult to assess because it is closely related to the gestational and postnatal age of the neonate as well as the cause of the thrombocytopenia and the severity of concurrent conditions. In practice, the decision is governed by two questions. When is it appropriate to administer platelets to non-bleeding patients (prophylactic transfusions)? When is it appropriate to administer platelets to actively bleeding patients? Our current guidelines (table 4) reflect our current practice, ${ }^{17}$ but, as with published consensus guidelines, are not based on good quality trial evidence.

\section{CLINICAL IMPACT OF NEONATAL THROMBOCYTOPENIA}

It is very difficult to critically assess the clinical impact of neonatal thrombocytopenia. Previous studies suggest that it is a risk factor for haemorrhage (particularly intraventricular haemorrhage), ${ }^{14}{ }^{15}{ }^{58-60}$ mortality, ${ }^{14-16}$ and adverse neurodevelopmental outcome. ${ }^{59}$ However, it is unclear whether thrombocytopenia itself directly contributes to adverse outcome or is simply a marker of the severity of precipitating complications-for example, perinatal asphyxia-which themselves carry a poor prognosis. One way to determine the clinical impact is to prevent thrombocytopenia by the use of platelet transfusion in a high risk population. In the only randomised trial designed to address this, Andrew et $a l^{61}$ found no benefit (reduction in haemorrhage) in preterm neonates when moderate thrombocytopenia (platelets $\left.(50-150) \times 10^{9} / 1\right)$ was prevented by platelet transfusion. Appropriate lower limit platelet counts for all groups of preterm neonates were not defined, as all neonates were deemed eligible for platelet transfusion if their platelet count fell below $50 \times 10^{9} /$. However, this study clearly defines a platelet count of $>50 \times$ $10^{9} / \mathrm{l}$ as a "safe" level, even in sick neonates (table 4 ).

\section{FUTURE DEVELOPMENTS}

Progress in the assessment and management of neonates who develop significant neonatal thrombocytopenia is likely to

\begin{tabular}{|c|c|c|c|}
\hline $\begin{array}{l}\text { Platelet count } \\
\left(\times 10^{9} / 1\right)\end{array}$ & Non-bleeding neonate & Bleeding neonate & NAITP (proven or suspected) \\
\hline $\begin{array}{l}<30 \\
30-49\end{array}$ & $\begin{array}{l}\text { Consider transfusion in all patients } \\
\text { Do not transfuse if clinically stable } \\
\text { Consider transfusion if: } \\
\text { - }<1000 \mathrm{~g} \text { and }<1 \text { week of age } \\
\text { - clinically unstable (e.g. fluctuating } \\
\text { blood pressure or perfusion) } \\
\text { - previous major bleeding (e.g. grade } \\
\text { 3-4 IVH or pulmonary haemorrhage) } \\
\text { - current minor bleeding (e.g. } \\
\text { petechiae, puncture site oozing or } \\
\text { blood stained ET secretions) } \\
\text { - concurrent coagulopathy } \\
\text { - requires surgery or exchange } \\
\text { transfusion }\end{array}$ & $\begin{array}{l}\text { Transfuse } \\
\text { Transfuse }\end{array}$ & $\begin{array}{l}\text { Transfuse (with HPA compatible platelets) } \\
\text { Transfuse (with HPA compatible platelets if any bleeding) }\end{array}$ \\
\hline $\begin{array}{l}50-99 \\
>99\end{array}$ & $\begin{array}{l}\text { Do not transfuse } \\
\text { Do not transfuse }\end{array}$ & $\begin{array}{l}\text { Transfuse } \\
\text { Do not transfuse }\end{array}$ & $\begin{array}{l}\text { Transfuse (with HPA compatible platelets if major bleeding present) } \\
\text { Do not transfuse }\end{array}$ \\
\hline
\end{tabular}

NAITP, Neonatal alloimmune thrombocytopenia; HPA, human platelet antigen; IVH, intraventricular haemorrhage; ET, endotracheal. 
require at least three different approaches: (a) improved diagnosis and treatment for the conditions precipitating severe thrombocytopenia (mainly sepsis and NEC); $(b)$ development of evidence based protocols for platelet transfusion in nonimmunologically mediated neonatal thrombocytopenia; (c) development of alternative treatments to stimulate platelet production (haemopoietic growth factor therapy).

\section{Improved diagnosis and treatment for conditions that cause severe thrombocytopenia}

Several approaches to prevention and treatment of the principal conditions that precipitate severe thrombocytopenia are currently under investigation in preterm infants, including granulocyte-macrophage colony stimulating factor, ${ }^{62}$ IVIG $^{63}$ and pentoxifylline ${ }^{64}$ to reduce the incidence of neonatal sepsis, and epidermal growth factor for the treatment of NEC. ${ }^{65}$

\section{Evidence based protocols for neonatal platelet transfusion}

Although difficult to achieve without putting neonates at risk of haemorrhage, there remains a fundamental need for randomised controlled trials to define the safe lower limit for platelet counts in sick newborns and to define which neonates will benefit from specific treatment of their thrombocytopenia. Platelet transfusion practice itself also deserves attention, as questions such as optimal platelet dose, interval between transfusions, and platelet monitoring after transfusion remain unanswered. Also, as with all other interventions in neonatal medicine, long term follow up of neonates who have received platelet transfusions is essential both to determine if they actually improve outcome but, equally importantly, to exclude the possibility of any unexpected long term adverse consequences.

\section{Haemopoietic growth factors}

As platelet underproduction causes or contributes to most episodes of neonatal thrombocytopenia, ${ }^{1621}$ thrombopoietic growth factors, such as Tpo and interleukin 11 (IL-11), may ameliorate thrombocytopenia and provide an alternative to the uncertainties surrounding platelet transfusion.

\section{Thrombopoietin}

This is the major regulator of platelet production in humans, including neonates. ${ }^{21} 2366$ Recombinant human (rh) Tpo stimulates megakaryocyte precursor and progenitor cells from term and preterm neonates. ${ }^{22} 2367-69$ However, recent evidence also shows that, although neonates of all gestational ages produce endogenous Tpo, they appear to produce suboptimal amounts of Tpo in response to thrombocytopenia, ${ }^{21}{ }^{23}$ suggesting that rhTpo may have a role in the treatment of neonatal thrombocytopenias. However, the clinical application of rhTpo seems likely to be limited by the development of neutralising antibodies and by the delay of six to seven days after administration before the platelet count rises, ${ }^{70}$ a lag time during which many neonatal thrombocytopenias will resolve.

\section{IL-1 1}

This stimulates platelet production from megakaryocytes and is now commercially available for this purpose. ${ }^{71}$ In animal models, IL-11 also conveys survival benefit during sepsis ${ }^{72-74}$ and bowel injury ${ }^{71}$ and has recently been shown to ameliorate NEC. ${ }^{75}$ Our work shows that rhIL-11 stimulates megakaryocytes from term and preterm neonates, ${ }^{76}$ and endogenous IL-11 levels are significantly raised in neonates with sepsis and NEC, ${ }^{77}{ }^{78}$ suggesting that IL- 11 is intimately involved in the pro/counterinflammatory cytokine response in these conditions. Therefore rhIL-11 treatment during neonatal sepsis and NEC would have the potential to ameliorate thrombocytopenia while also benefiting the underlying conditions. Although to date there have been no reports of the use of
rhIL- 11 in the treatment of neonates with thrombocytopenia and or sepsis/NEC, this remains an exciting prospect for the future.

\section{CONCLUSION}

Neonatal thrombocytopenia is a common clinical problem. Fortunately most episodes are mild or moderate and resolve spontaneously without apparent clinical sequelae. For more severe episodes, the recent demonstration of impaired megakaryocytopoiesis and platelet production as a major contributor to neonatal thrombocytopenia is an important advance both for our understanding of the underlying disease processes and the potential for innovative treatment. The use of haemopoietic growth factors to stimulate neonatal platelet production, and particularly the potential of IL-11, is an exciting prospect. At a more basic level, however, there remains a need for trials to define the safe lower limit for platelet counts in sick newborns and to define which neonates will benefit from treatment of their thrombocytopenia. Finally, ensuring accurate diagnosis and determining effective fetal and neonatal treatment for NAITP, the thrombocytopenia currently identifiable as directly causing most mortality and morbidity, should remain a goal for all fetal medicine specialists, haematologists, and neonatal paediatricians.

\section{Authors' affiliations}

I Roberts, N A Murray, Imperial College, Hammersmith Campus, London W12 ONN, UK

\section{REFERENCES}

1 Blajchman MA, Goldman M. Bacterial contamination of platelet concentrates: incidence, significance, and prevention. Semin Hematol 2001;38(suppl 11):20-6.

2 Regan F, Taylor C. Blood transfusion medicine. BM 2002;325: 143-7.

3 Pahal G, Jauniaux E, Kinnon C, et al. Normal development of human fetal hematopoiesis between eight and seventeen weeks' gestation. Am J Obstet Gynecol 2000;183: 1029-34.

4 Holmberg L, Gustavii B, Jonsson A. A prenatal study of fetal platelet count and size with application to the fetus at risk of Wiskott Aldrich syndrome. J Pediatr 1983:102:773-81.

5 Forestier F, Daffos F, Galacteros F. Haematological values of 163 normal fetuses between 18 and 30 weeks of gestation. Pediatr Res 1986;20:342-6.

6 Forestier F, Daffos F, Catherine N, et al. Developmental hematopoiesis in normal human fetal blood. Blood 1991;77:2360-3.

7 Hohlfeld P, Forestier F, Kaplan C, et al. Fetal thrombocytopenia: a retrospective survey of 5,194 fetal blood samplings. Blood 1994;84:1851-6.

8 Burrows RF, Kelton JG. Incidentally detected thrombocytopenia in healthy mothers and their infants. N Engl J Med 1988;319:142-5.

9 Sainio S, Jarvenpaa A-S, Renlund M, et al. Thrombocytopenia in term infants: a population-based study. Obstet Gynecol 2000;95:441-6.

10 de Moerloose P, Boehlen F, Extermann P, et al. Neonatal thrombocytopenia: incidence and characterization of maternal antiplatelet antibodies by MAIPA assay. Br J Haematol 1998; 100:735-40.

11 Burrows RF, Kelton JG. Fetal thrombocytopenia and its relation to maternal thrombocytopenia. N Engl J Med 1993;329:1463-6.

12 Uhrynowska $M$, Niznikowska-Marks M, Zupanska B. Neonatal and maternal thrombocytopenia: incidence and immune background. Eur J Haematol 2000;64:42-6.

13 Dreyfus $M$, Kaplan C, Verdy E, et al. Frequency of immune thrombocytopenia in newborns: a prospective study. Immune Thrombocytopenia Working Group. Blood 1997;89:4402-6.

14 Castle V, Andrew M, Kelton J, et al. Frequency and mechanism of neonatal thrombocytopenia. J Pediatr 1986;108:749-55.

15 Mehta P, Rohitkumar V, Neumann L, et al. Thrombocytopenia in the high risk infant. J Pediatr 1980;97:791-4.

16 Murray NA, Roberts IAG. Circulating megakaryocytes and their progenitors in early thrombocytopenia in preterm neonates. Pediatr Res 1996;40: 112-19.

17 Murray NA, Howarth L, McCloy MP, et al. Platelet transfusion in the management of severe thrombocytopenia in neonatal intensive care unit (NICU) patients. Transfus Med 2002;12:35-41.

18 Homans A. Thrombocytopenia in the neonate. Pediatr Clin North Am 1996;43:737-56.

19 Blanchette VS, Rand ML. Platelet disorders in newborn infants: diagnosis and management. Semin Perinatol 1997;21:53-62.

20 Sola MC, Del Vecchio A, Rimsza LM. Evaluation and treatment of thrombocytopenia in the neonatal intensive care unit. Clin Perinatol 2000;27:655-79 
21 Sola MC, Calhoun DA, Hutson AD, et al. Plasma thrombopoietin concentrations in thrombocytopenic and non-thrombocytopenic patients in a neonatal intensive care unit. Br J Haematol 1999; 104:90-2.

22 Murray NA, Watts TL, Roberts IAG. Endogenous thrombopoietin levels and effect of recombinant human thrombopoietin on megakaryocyte precursors in term and preterm babies. Pediatr Res 1998;43:148-51.

23 Watts TL, Murray NA, Roberts IAG. Thrombopoietin has a primary role in the regulation of platelet production in preterm babies. Pediatr Res 1999;46:28-32

24 Castle V, Coates G, Kelton JG, et al. ${ }^{111}$ In-oxine platelet survivals in thrombocytopenic infants. Blood 1987;70:652-6.

25 Forestier F, Hohlfeld P. Management of fetal and neonatal alloimmune thrombocytopenia. Biol Neonate 1998;74:395-401.

26 Muellar-Eckhardt C, Kiefel V, Grubert A, et al. 348 cases of suspected neonatal alloimmune thrombocytopenia. Lancet 1989;i:363-6.

27 Williamson LM, Hackett G, Rennie J, et al. The natural history of fetomaternal alloimmunization to the platelet-specific antigen HPA-la (PIA 1, Zwa) as determined by antenatal screening. Blood 1998;92:2280-7.

28 Davoren A, McParland P, Barnes CA, et al. Neonatal alloimmune thrombocytopenia in the Irish population: a discrepancy between observed and expected cases. J Clin Pathol 2002;55:289-92.

29 Glade-Bender J, McFarland JG, Kaplan C, et al. Anti-HPA-3A induces severe neonatal alloimmune thrombocytopenia. J Pediatr 2001;138:862-7.

30 De Tar MW, Klohe E, Grosset A, et al. Neonatal alloimmune thrombocytopenia with HLA alloimmunization: case report with immunohematologic and placental findings. Pediatr Dev Pathol 2002; 5:200-5

31 Sasaki M, Yagihashi A, Kobayashi D, et al. Neonatal alloimmune thrombocytopenia due to anti-human leukocyte antigen antibody: a case report. Pediatr Hematol Oncol $2001 ; 18: 519-24$.

32 Grainger JD, Morrell G, Yates J, et al. Neonatal alloimmune thrombocytopenia with significant HLA antibodies. Arch Dis Child Fetal Neonatal Ed 2002:86:F200-1.

33 Sharif U, Kuban K. Prenatal intracranial hemorrhage and neurologic complications in alloimmune thrombocytopenia. J Child Neuro $2001 ; 16: 838-42$.

34 Jaegtvik S, Husebekk A, Aune B, et al. Neonatal alloimmune thrombocytopenia due to anti-HPA la antibodies: the level of maternal antibodies predicts the severity of thrombocytopenia in the newborn. $\mathrm{Br}$ J Obstet Gynaecol 2000; 107:691-4.

35 Ouwehand WH, Smith G, Ranasinghe E. Management of severe alloimmune thrombocytopenia in the newborn. Arch Dis Child Fetal Neonatal Ed 2000:82:F173-5.

36 Radder CM, Brand A, Kanhai HH. A less invasive treatment strategy to prevent intracranial hemorrhage in fetal and neonatal alloimmune thrombocytopenia. Am J Obstet Gynecol 2001;185:683-8

37 Kaplan C. Alloimmune thrombocytopenia of the fetus and the newborn. Blood Rev 2002;16:69-72

38 Rothenberger S. Neonatal alloimmune thrombocytopenia. Ther Apher 2002;6:32-5

39 Jolly MC, Letsky EA, Fisk NM. The management of fetal alloimmune thrombocytopenia. Prenat Diagn 2002;22:96-8.

40 Bussel JB. Alloimmune thrombocytopenia in the fetus and newborn. Semin Thromb Hemost 2001;27:245-52.

41 Ahya R, Turner ML, Urbaniak SJ. Fetomaternal alloimmune thrombocytopenia. Transfus Apheresis Sci 2001;25:139-45.

42 Murphy MF, Waters AH, Doughty HA, et al. Antenatal management of fetomaternal alloimmune thrombocytopenia: report of 15 affected pregnancies. Transfus Med 1994;4:281-92.

43 Overton TG, Duncan KR, Jolly $M$, et al. Serial aggressive platelet transfusion for fetal alloimmune thrombocytopenia: platelet dynamics and perinatal outcome. Am J Obstet Gynecol 2002;186:826-31.

44 Silver RM, Porter TF, Branch DW, et al. Neonatal alloimmune thrombocytopenia: antenatal management. Am J Obstet Gynecol 2000;182:1233-8.

45 Kaplan C, Murphy MF, Kroll H, et al. Feto-maternal alloimmune thrombocytopenia: antenatal therapy with lvlgG and steroids: more questions than answers. European Working Group on FMAIT. Br J Haematol 1998; 100:62-5.

46 Sainio S, Teramo K, Kekomaki R. Prenatal treatment of severe fetomaternal alloimmune thrombocytopenia. Transfus Med 1999:9:321-30.

47 Blanchette VS, Johnson J, Rand M. The management of alloimmune neonatal thrombocytopenia. Baillieres Clin Haematol 2000;13:365-90.

48 Bussel J, Kaplan C. The fetal and neonatal consequences of maternal alloimmune thrombocytopenia. Baillieres Clin Haematol 1998;11:391-408.

49 Ranasinghe E, Walton JD, Hurd CM, et al. Provision of platelet support for fetuses and neonates affected by severe fetomaternal alloimmune thrombocytopenia. Br J Haematol 2001;1 13:40-2

50 Lee K, Beaujean F, Bierling P. Treatment of severe fetomaternal alloimmune thrombocytopenia with compatible frozen-thawed platelet concentrates. Br J Haematol 2002;117:482-3.

51 Bussel JB. Immune thrombocytopenia in pregnancy: autoimmune and alloimmune. J Reprod Immunol 1997;37:35-61.
52 Valat AS, Caulier MT, Devos $P$, et al. Relationships between severe neonatal thrombocytopenia and maternal characteristics in pregnancies associated with autoimmune thrombocytopenia. Br J Haematol 1998; 103:397-401.

53 Kelton JG. Idiopathic thrombocytopenic purpura complicating pregnancy. Blood Rev 2002;16:43-6.

54 Burrows RF, Kelton JG. Low fetal risks in pregnancies associated with idiopathic thrombocytopenic purpura. Am J Obstet Gynecol 1990; 163:1 147-50.

55 Voak D, Cann R, Finney RD, et al. Working party: Gibson B, Hann IM, Holland B, Letsky EA, Napier JAF, de Silva M, Wardrop CA, Webb MSC. Guidelines for administration of blood products: transfusion of infants and neonates. Transfus Med 1994;4:63-9.

56 Roberts IAG, Murray NA. Management of thrombocytopenia in neonates. Br J Haematol 1999; 105:864-70

57 Calhoun DA, Christensen RD, Edstrom CS, et al. Consistent approaches to procedures and practices in neonatal hematology. Clin Perinatol 2000:27:733-53.

58 Amato M, Fauchere JC, Herman Jr U. Coagulation abnormalities in low birth weight infants with peri-intraventricular hemorrhage. Neuropediatrics 1988;19:154-7.

59 Andrew M, Castle V, Saigal S, et al. Clinical impact of neonatal thrombocytopenia. J Pediatr 1987; 1 10:457-64

60 Van De Bor M, Briet E, Van Bel F, et al. Hemostasis and periventricular-intraventricular hemorrhage of the newborn. American Journal of Diseases of Children 1986;140:1131-4.

61 Andrew M, Vegh P, Caco VC, et al. A randomized, controlled trial of platelet transfusions in thrombocytopenic premature infants. J Pediatr 1993; 123:285-91.

62 Carr R, Modi N, Dore CJ, et al. A randomized, controlled trial of prophylactic granulocyte-macrophage colony-stimulating factor in human newborns less than 32 weeks gestation. Pediatrics 1999:103:796-802.

63 Haque KN. Intravenous immunoglobulins versus sepsis. Pediatrics 2000;105: 1173

64 Lauterbach R, Pawlik D, Kowalczyk D, et al. Effect of the immunomodulating agent, pentoxifylline, in the treatment of sepsis in prematurely delivered infants: a placebo-controlled, double-blind trial. Crit Care Med 1999:27:807-14.

65 Fagbemi AO, Wright N, Lakhoo K, et al. Immunoreactive epidermal growth factor receptors are present in gastrointestinal epithelial cells of preterm infants with necrotising enterocolitis. Early Hum Dev 2001;65:1-9.

66 Albert TSE, Meng G, Simms P, et al. Thrombopoietin in the thrombocytopenic term and preterm newborn. Pediatrics 2000;105:1286-91.

67 Nishihira H, Toyoda Y, Miyazaki H, et al. Growth of macroscopic human megakaryocyte colonies from cord blood with recombinan human thrombopoietin (c-mpl ligand) and the effects of gestational age on the frequency of colonies. Br J Haematol 1996;92:23-8.

68 Sola MC, Du Y, Hutson AD, et al. Dose-response relationship of megakaryocyte progenitors from bone marrow and peripheral blood of thrombocytopenic neonates to recombinant thrombopoietin [abstract]. Pediatr Res 1999:45:153A.

69 Watts TL, Murray NA, Roberts IAG. Thrombopoietin in term and preterm babies with and without thrombocytopenia [abstract]. Blood 1997;90:141a.

70 Sola MC, Christensen RD, Hutson AD, et al. Pharmacokinetics, pharmacodynamics, and safety of administering pegylated recombinant megakaryocyte growth and development factor to newborn rhesus monkeys. Pediatr Res 2000:47:208-14

71 Schwertschlag US, Trepicchio WL, Dykstra KH, et al. Hemopoietic, immunomodulatory and epithelial effects of interleukin-11. Leukemia 1999;13:1307-15.

72 Chang $M$, Williams A, Ishizawa $L$, et al. Endogenous interleukin-11 (IL-1 1) expression is increased and prophylactic use of exogenous IL-1 enhances platelet recovery and improves survival during thrombocytopenia associated with experimental group B sepsis in neonatal rats. Blood Cells Mol Dis 1996;22:57-67.

73 Opal SM, Jhung JW, Keith JC Jr, et al. Recombinant human interleukin-1 1 in experimental pseudomonas aeruginosa sepsis in immunocompromised animals. J Infect Dis 1998;178:1205-8.

74 Opal SM, Jhung JW, Keith JC Jr, et al. Additive effects of human recombinant interleukin-11 and granulocyte colony-stimulating factor in experimental gram-negative sepsis. Blood 1999:93:3467-72.

75 Claud E, Jackson $M$, Jilling $T$, et al. Interleukin-1 1 diminishes intestina injury in a rat model of necrotizing enterocolitis [abstract]. Pediatr Res 2000;47:163A

76 McCloy MP, Howarth L, Watts TL, et al. The role of IL-1 1 in neonatal thrombocytopenia. Hematol J 2000;1 (suppl 1):139.

77 McCloy MP, Howarth L, Watts TL, et al. The role of IL-1 1 in neonatal thrombocytopenia [abstract]. Blood 2000;96(suppl 1):564a.

78 McCloy MP, Roberts IAG, Howarth L, et al. IL-1 1 levels in healthy and thrombocytopenic neonates: potential for IL-1 1 therapy in sepsis- and NEC-related thrombocytopenia in preterm neonates. Pediatr Res 2002;51:756-60.

79 Roberts IAG, Murray NA. Thrombocytopenia in the newborn. In: Michelson A, ed. The platelets. New York: Elsevier Science, 2002:635-8 